\title{
The early management of meningococcal disease
}

\author{
T J Hodgetts, A Brett, N Castle
}

Neisseria meningitidis is an important cause of bacterial meningitis, particularly in infants over three months old and in teenagers. The relative importance of meningococcal disease over invasive Haemophilus influenzae disease has increased following the introduction of $\mathrm{Hib}$ capsular vaccines in 1990. In about one fifth of infections with Neisseria meningitidis the organism causes a predominantly septicaemic illness. ${ }^{1}$ Meningococcal meningitis and meningococcal septicaemia together constitute the spectrum of meningococcal disease. The overall mortality of meningococcal disease is around $8 \%$ (up to $5 \%$ in meningitis, rising to $15-20 \%$ with septicaemia), which is improved by the early administration of antibiotics. ${ }^{1-3}$ The infection is characteristically fulminant, with rapid clinical deterioration and death in a matter of hours - the mortality is particularly high in meningococcal septicaemia when there has been a delay in diagnosis or treatment (up to $60 \%$ when there is septic shock ${ }^{3}$ ). Therefore early recognition and aggressive management will improve the outcome in meningococcal disease.

Nationally there has been an increasing trend in laboratory confirmed meningococcal disease over the 10 years from 1985 to 1995 (Public Health Laboratory Service meningococcal reference laboratory, personal communication; fig 1), although it is recognised that there is a consistent underreporting of survivors of meningococcal disease-perhaps because of failure to confirm the diagnosis bacteriologically. $^{2}$ The importance of this infection at a community and district general hospital level has prompted the development of a treatment algorithm for prehospital and hospital use, to encourage the early aggressive treatment of this disease.

In this paper we review the current recom-

Accident and

Emergency

Department, Frimley

Park Hospital NHS

Trust, Portsmouth

Road, Camberley,

Surrey GU16 5UJ, UK

T J Hodgetts

A Brett

N Castle

Correspondence to: Major T J Hodgetts.

email: tim@blenheim. softnet.co.uk

Accepted for publication 21 July 1997

It is important to recognise that there is a difference between meningococcal meningitis presentation and management. These conditions are not, however, always clearly differentiated. The danger arises when both are collec- tively referred to as "meningococcal meningitis," with the subsequent failure to anticipate the development of septic shock, and the failure to direct treatment towards preventing the progress of shock.

Meningococcal meningitis characteristically manifests with fever, severe headache, vomiting, neck stiffness with positive Kernig's sign, photophobia, drowsiness, and confusion. In the very young the principal indicators will be fever, vomiting, drowsiness, poor feeding, and irritability. ${ }^{4}$

The characteristic feature of meningococcal septicaemia is a purpuric rash (fig 2) in a patient who is febrile and tachycardic. A purpuric rash in an unwell child should be treated as meningococcal septicaemia until proven otherwise. Signs of circulatory shock often develop rapidly with tachypnoea, tachycardia, cold peripheries, and oliguria. Drowsiness, and confusion in older children, are late signs. Limb pain (venous thrombosis or arthritis) may be a presenting feature.

\section{Prehospital management}

In all cases the priorities are to secure and maintain a clear airway, to give supplemental high concentration oxygen, and to support ventilation where needed. Rapid transport to hospital is essential.

Parenteral benzylpenicillin given by the general practitioner is associated with a significant reduction in mortality from meningococcal disease. $^{23}$ It is preferable to give this intravenously, but the intramuscular or intraosseous route are acceptable alternatives. The dose for meningococcal disease is $300 \mathrm{mg}$ for infants, $600 \mathrm{mg}$ for one to nine year olds, and $1200 \mathrm{mg}$ for those of 10 years or more. There is no evidence to support the prehospital use of corticosteroids. ${ }^{3}$ In some circumstances the general practitioner may be involved in more extensive resuscitation, perhaps in rural areas when the prehospital time is prolonged and where the doctor has received extended practical skills training on an advanced paediatric life support $^{5}$ or paediatric advanced life support ${ }^{6}$ course. In this case the intraosseous route should be considered when intravenous access is difficult and fluid is required in addition to the antibiotics. and meningococcal septicaemia, both in their

\section{Accident and emergency management}

The immediate management is again directed towards the support of the airway, breathing, 


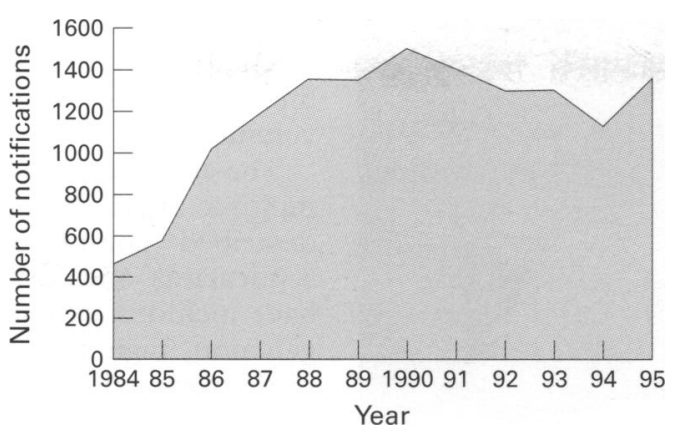

Figure 1 Laboratory confirmed meningococcal disease in England and Wales, 1984-1995. (Source: Public Health Laboratory Service reference laboratory.)

and circulation. ${ }^{5}$ Cardiorespiratory failure is a common feature of meningococcal septicaemic shock; high concentrations of oxygen should be given to all patients, and early ventilation, initially with $100 \%$ oxygen, is often required. Do not wait for respiratory failure to develop when you are treating septicaemic shock: acute lung injury is common, and early elective ventilation is indicated.

Vomiting should be anticipated and consideration given to electively intubating and ventilating any patient with a reduced level of response, in order to protect the airway. An intragastric tube should otherwise be placed early in the spontaneously breathing patient.

Hypovolaemia is consistently present in meningococcal septicaemia, as a result of the widespread increase in vascular permeability. Repeated boluses of fluid are needed to restore the peripheral circulation. Colloid is preferred because the fluid lost is protein rich, and $4.5 \%$ human albumin solution is preferred to the synthetic colloids. Fluid is given in $20 \mathrm{ml} / \mathrm{kg}$ boluses, the weight of the child being estimated in one of the following ways: (1) asking a parent; (2) by relating it to the child's length on a Broselow tape or paediatric resuscitation chart $^{7}$; (3) by using the formula weight $(\mathrm{kg})=$ [age in years +4$] \times 2$, which is accurate from one to 10 years.

In the septicaemic child who requires immediate fluid resuscitation there should be no delay in inserting an intraosseous needle if two attempts at peripheral venous cannulation fail, or more than 90 seconds elapse. ${ }^{5}$ Fluids and drugs are then best given by syringe and a short connection tube (the connection tube prevents repeated direct pressure on the needle). It is wise to protect this line, for example in a box splint. The intraosseous route is generally recommended for children aged six years or less.

Cold extremities, a delayed capillary refill time, a rising pulse and respiratory rate, confusion, and a poor urine output are all indicators of developing septic shock and inadequate resuscitation of the circulation. Hypotension is a late sign in children, and when it occurs it is often precipitous.

The traditional antibiotic of choice for Neisseria meningitidis is benzylpenicillin. Penicillin resistant meningococci have been recognised in Spain and South Africa, and a small number of penicillin resistant isolates have been identified in the United Kingdom. ${ }^{8}$ For this reason, and because other pathogens may commonly produce a meningitis picture (and on occasion a septicaemic picture), a broad spectrum third generation cephalosporin is recommended as first line antimicrobial treatment. Additional penicillin is not required. ${ }^{4-11}$ Either cefotaxime $200 \mathrm{mg} / \mathrm{kg} /$ day in three or four divided doses, or ceftriaxone $80 \mathrm{mg} / \mathrm{kg} /$ day in a single daily dose (as it has a much longer half life) are suitable. ${ }^{49}$ The single daily dose of ceftriaxone is attractive, but it is associated with a higher incidence of diarrhoea. It has also been recommended that the first dose of antibiotics is increased by $50 \%$, to ensure a high initial serum and CSF concentration. ${ }^{12}$

For meningitis and septicaemia in infants one to three months old additional ampicillin $200 \mathrm{mg} / \mathrm{kg} / \mathrm{day}$ is recommended, ${ }^{811}{ }^{13}$ to ensure activity against an increased pathogenic spectrum which includes Escherichia coli, Listeria monocytogenes, and salmonella species. ${ }^{10} 14$

Hypoglycaemia is common in any child who is seriously unwell, and must be anticipated in the septicaemic child. Capillary blood glucose estimates should be performed regularly, and hypoglycaemia corrected when values fall below $2.0 \mathrm{mmol} /$ litre. A range of electrolyte disturbances can also occur (hypocalcaemia, hypokalaemia, hypomagnesaemia, hypophosphataemia) and these should be tested for and corrected where appropriate. Blood should also be taken for a clotting screen and cross match, and blood gas analysis can be used as an adjunct to monitor the effectiveness of resuscitation. Overt coagulopathy may be identified by bleeding from venepuncture sites, necessitating fresh frozen plasma. Anaemia can be profound and blood may be required after the initial colloid resuscitation.

The evidence for the benefit of dexamethasone in the management of meningitis is far from conclusive, although the drug has been in regular use for several years. ${ }^{1015}$ The current recommendation of the infectious diseases committee of the American Academy of Pediatrics is to use dexamethasone $(0.15$ $\mathrm{mg} / \mathrm{kg}$ six hourly ${ }^{14}$ ) for the treatment of meningitis caused by $H$ influenzae, and simply to consider its use for meningitis of unknown origin, or known to be caused by $N$ meningitidis or $S$ pneumoniae. ${ }^{16}$ Accident and emergency clinicians should seek the advice of medical staff from the admitting paediatric intensive care unit with respect to the use of steroids.

\section{Confirming the diagnosis}

The diagnosis is largely a clinical one. On arrival in hospital venous blood should be taken for blood cultures, but the yield will be reduced by the benzylpenicillin given by the general practitioner. Lumbar puncture should be considered in patients with meningitis, but not those with septicaemia (because it will not alter clinical management, and the cerebrospinal fluid (CSF) is likely to be sterile) or evidence of raised intracranial pressure (because it may produce cerebral herniation). ${ }^{11}$ The diagnosis may also be confirmed by culture from a throat swab or rash aspirate, antigen detection, serodiagnosis, and polymer- 


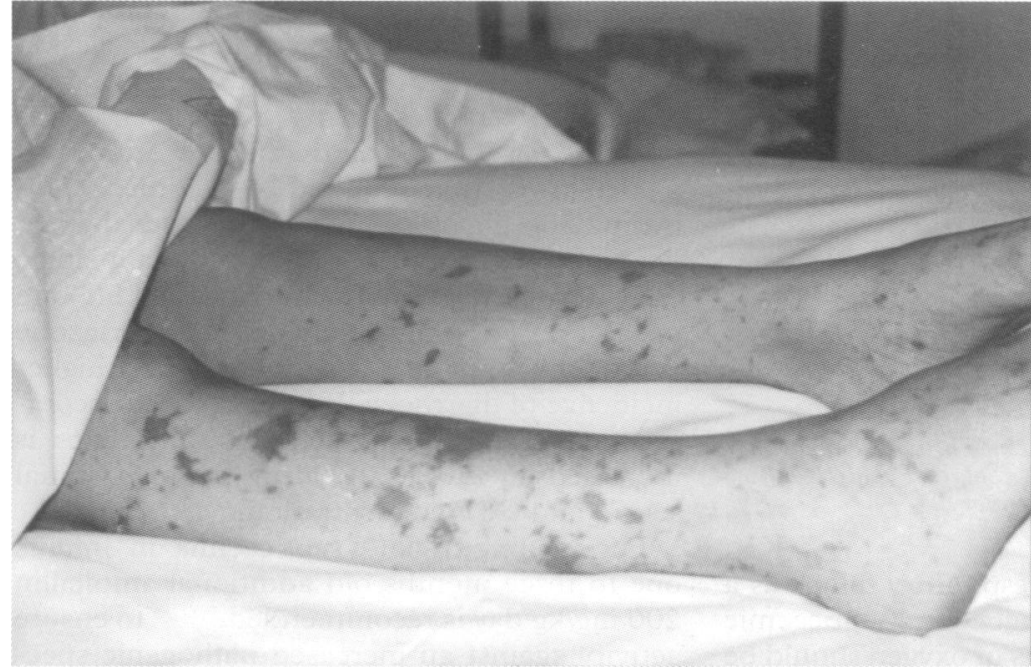

Figure 2 Purpuric rash of meningococcal septicaemia.

ase chain reaction testing of blood or $\mathrm{CSF}^{3}$ which will not be affected by the administration of antibiotics.

There is no role for computerised tomography in the initial management of patients with life threatening meningococcal disease.

\section{Definitive care}

The further management of patients suffering from meningococcal disease must address the multisystem nature of the illness. Patients with meningitis and no depression of conscious level may be managed on a general ward. However, they must be assessed repeatedly, as deterioration may be sudden and catastrophic. Indications for elective intubation and ventilation are potential airway compromise, severe agitation, recurrent or refractory seizures, and impending or established respiratory failure.

Children who have been intubated, who have shock or evidence of disseminated intravascular coagulopathy (including rapidly progressive purpuric rash), or who have metabolic acidosis should be referred to a specialist paediatric intensive care unit (PICU). Adults requiring continuing respiratory or cardiovascular support are managed on a general intensive care unit. Prognostic indicators and scoring systems have been used to predict disease progression, and the requirement for specialist care, based on clinical and laboratory information. ${ }^{4} 17$

There are 15 paediatric intensive care retrieval teams in the United Kingdom (telephone survey of all PICUs listed in the 1996 Directory of emergency and special care units ${ }^{18}$ ) and early contact by the referring district general hospital is to be recommended where this service is available.

Those children with meningococcal septicaemia and haemodynamic instability should at first receive aggressive fluid resuscitation. Hypotension is the result of hypovolaemia, dysregulation of vascular tone, and myocardial dysfunction-inotropic support is very often required. Dobutamine, low dose dopamine, and adrenaline are used, but practice varies throughout the country. An initial combination of dobutamine and low dose dopamine are rec- ommended when $40 \mathrm{ml} / \mathrm{kg}$ of colloid has been given. Insertion of a central venous pressure line will provide vital information concerning volume status.

The acute respiratory distress syndrome and multiple organ dysfunction syndrome are well described complications of meningococcal septicaemia, and advanced treatment strategies have included extracorporeal membrane oxygenation, high frequency jet ventilation, and nitric oxide, with variable degrees of success in both children $^{1920}$ and adults. ${ }^{21}$

\section{The algorithm}

The algorithm is presented as a double sided A4 sheet for rapid reference (fig 3), and is divided into three sections: recognition, immediate actions, and specific treatment. It is intended to be used by ambulance staff, general practitioners, accident and emergency clinicians, and paediatricians in the early management of meningitis and meningococcal septicaemia. The principal objective is to deliver an appropriately resuscitated patient to the definitive care facility (ward or intensive care unit). The algorithm does not attempt to dictate the policy of a specialist intensive care unit.

\section{RECOGNITION}

Meningitis is recognised by the history and examination (as described above). The underlying causative organism is not important initially, as all cases of meningitis should be treated in the same manner. Meningococcal septicaemia will be suspected in a febrile child who is shocked and who has a petechial rash.

\section{IMMEDIATE ACTIONS}

The objectives of the ambulance service are:

- To maintain and secure a clear airway;

- To provide supplemental high concentration oxygen, and to assist ventilation if needed;

- To provide rapid transport to hospital;

- To warn the accident and emergency department in advance.

The need for antibiotic prophylaxis for the ambulance crew is a common concern: it is not required unless mouth to mouth ventilation has been given.

The general practitioner has an important role to give benzylpenicillin:

- Intravenous, intraosseous, or intramuscular benzylpenicillin, $300 \mathrm{mg}<1$ year, $600 \mathrm{mg}$ one to nine years, $1200 \mathrm{mg} \geqslant 10$ years.

The accident and emergency department should assemble a resuscitation team, to include an experienced anaesthetist and paediatrician, and follow these steps:

- Give supplemental high concentration oxygen, and consider intubation and ventilation;

- Obtain blood cultures (and other venous samples) when intravenous access is established;

- Give cefotaxime OR ceftriaxone intravenously; 

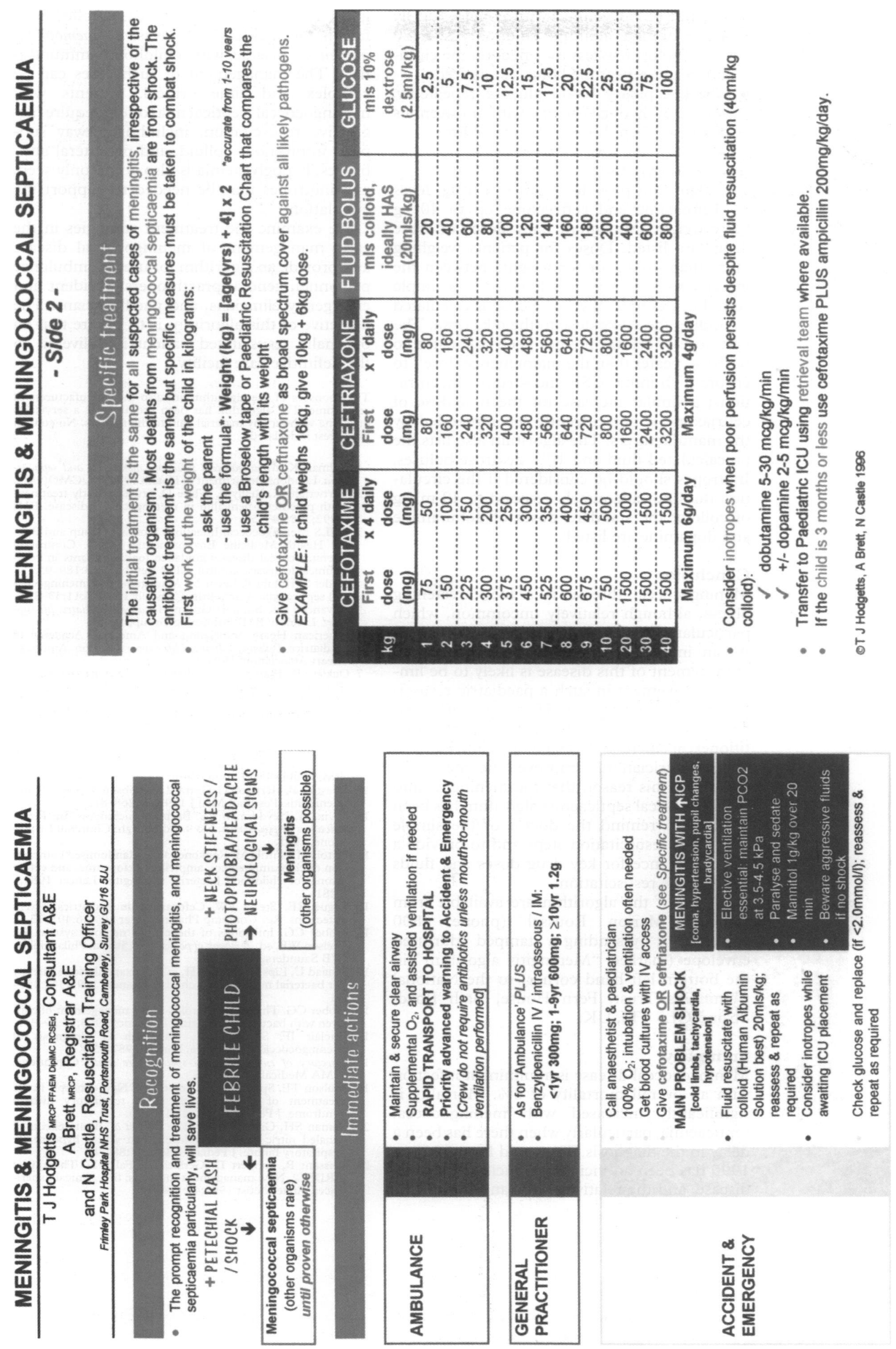

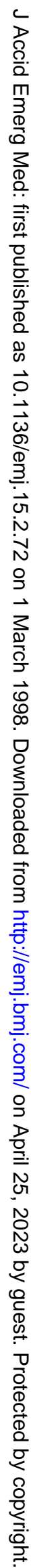

Figure 3 Treatment algorithm for meningococcal disease. 
- Expand blood volume with colloid, ideally $4.5 \%$ human albumin solution, as a 20 $\mathrm{ml} / \mathrm{kg}$ bolus; reassess the effect on the circulation and repeat the bolus as required;

- Check capillary blood glucose repeatedly, and treat hypoglycaemia with intravenous dextrose when this is $<2.0 \mathrm{mmol} /$ litre.

\section{SPECIFIC TREATMENT}

The algorithm provides a table of drug doses for cefotaxime and ceftriaxone up to $40 \mathrm{~kg}$ body weight. Maximum daily doses of these drugs are listed. Doses for patients weighing more than $40 \mathrm{~kg}$, or for weights between the increments of $10 \mathrm{~kg}$, are calculated by a simple sum. For example, the $16 \mathrm{~kg}$ dose is calculated by adding the $10 \mathrm{~kg}$ dose to the $6 \mathrm{~kg}$ dose. The first dose of cefotaxime has been calculated to be $50 \%$ greater than the maintenance $\operatorname{dose}^{13}$ to ensure high initial CSF and serum concentrations; a similar increase for the first dose of ceftriaxone is not currently recommended by the manufacturers. The algorithm also lists the precalculated fluid and $10 \%$ dextrose boluses. Inotropes should be considered if the circulation does not adequately respond to $40 \mathrm{ml} / \mathrm{kg}$ of colloid: the standard doses of dobutamine and dopamine are listed.

\section{Conclusion}

Meningococcal disease is a life threatening illness, although relatively uncommon, which particularly affects children and adolescents. As an individual doctor's experience in the management of this disease is likely to be limited, involvement in such a paediatric resuscitation will be stressful. However, early and aggressive treatment by the general practitioner, accident and emergency clinician, and the paediatrician may improve outcome.

It is for this reason that the meningitis and meningococcal septicaemia algorithm has been devised, to remind the doctor of the simple important resuscitation steps and to provide a rapid reference for key drug doses and fluids given in the resuscitation.

Copies of the algorithm are available from Hoechst Marion Roussel (phone 0800 282833), or by sending a stamped addressed envelope (marked "Meningitis algorithm" in the bottom left hand corner) to the National Meningitis Trust, Fern House, Bath Road, Stroud GL5 3TJ, UK.

\section{Summary}

Meningococcal disease is a fulminant infection with an overall mortality of $8 \%$. Mortality is significantly increased with meningococcal septicaemia, particularly when there has been a delay in the diagnosis. The trend from 1985 to 1995 has been an increase in incidence of this disease, and the relative importance of menin- gococcal disease has also increased following a fall in the incidence of invasive Haemophilus influenzae disease with childhood immunisation. The management of such cases can be complex and time critical. Patients with meningococcal septicaemia often require aggressive resuscitation, including airway support, intravenous colloid, and parenteral antibiotics; hypoglycaemia is also commonly seen, and inotropes may be needed to support the circulation.

We examine the treatment strategies in the early management of meningococcal disease and provide an algorithm for use by ambulance personnel, general practitioners, accident and emergency clinicians, and paediatricians. The objective of this algorithm is to ensure that an optimally resuscitated patient is delivered to the definitive care facility.

The sponsors of the algorithm are also the manufacturers of cefotaxime. The algorithm has been produced as a service to medicine without any material gain to the authors. No conflict of interest is declared.

1 Calman K. Meningococcal infection: meningitis and septicae mia. London: Department of Health 1994, PL/CMO(94)2 2 Cartwright K, Reilly S, White D, Stuart J. Early treatmen with parenteral penicillin in meningococcal disease. BM] 1992;305:143-52.

3 PHLS Meningococcal Infections Working Group and Public Health Medicine Environmental Group. Control of meningococcal disease: guidance for consultants in commeningococcal disease: guidance for consultants in com-

4 Nadel S, Habibi P, Levin M. Management of meningococcal septicaemia. Care of the Critically Ill 1995;11:33-8.

5 Advanced Life Support Group. Advanced paediatric life support. London: BMJ Publishing Group, 1993.

6 American Heart Association and American Academy of Pediatrics. Pediatric advanced life support. Boston: American Heart Association, 1994.

7 Oakley P, Phillips B, Molyneux E, Mackway-Jones K. Paediatric resuscitation. Updated standard reference chart. BMJ 1993;306:1096-8.

8 Klein N, Heyderman R, Levin M. Antibiotic choices for meningitis beyond the neonatal period. Arch Dis Child 1992;67:157-61

$9 \mathrm{BMA}$ and Royal Pharmaceutical Society of Great Britain British national formulary, No 31: March 1996. London BMA Publishing Group, 1996.

10 Priestley B, Harrison C, Gerrard M, Gibson A. Steroids and meningitis. Postgrad Med J 1993;69:268-81.

11 Levin M, Heyderman RS. Bacterial meningitis. In: Recent advances in paediatrics, No 9. Edinburgh: Churchill Livingstone, 1991 .

12 Peltola H, Anttila M, Renkonen O-V. Randomised comparison of chloramphenicol, ampicillin, cefotaxime, and ceftrison of chloramphenicol, ampicillin, cefotaxime, and ceftri1281-7.

13 Bégué RE, Steele RW. Ceftriaxone in pediatrics: severe infections. Rev Contemp Pharmacother 1995;6:401-13.

14 Prober CG. Infections of the central nervous system. In Nelson WE, ed. Textbook of pediatrics, 15 th ed. Philadelphia WB Saunders, 1996.

15 Schaad U, Lips U, Gnehm H, et al. Dexamethasone therapy for bacterial meningitis in children. Lancet 1993;342:45761 .

16 Prober CG. The role of steroids in the management of children with bacterial meningitis. Pediatrics 1995;95:29-31.

17 Sinclair JF, Skeoch CH, Hallworth D. Prognosis of meningococcal septicaemia. Lancet 1987;ii:38.

18 Directory of emergency and special care units. Cambridge: CMA Medical Data, 1996.

19 Paulson TE, Spear RM, Peterson BM. New concepts in the treatment of children with acute respiratory distress treatment of children with acute resp
syndrome. J Pediatr 1995;127:163-75.

20 Abman SH, Griebel JL, Parker DK, et al. Acute effects of inhaled nitric oxide in children with severe hypoxaemic inhaled nitric oxide in children with severe

21 Roissant R, Pappert D, Gerlach H, Falke K. Therapy of Roissant $R$, Pappert $D$, Gerlach $H$, Falke $K$. Therapy of
ARDS. 2. New management methods: first clinical experiences. Anaesthetist 1994;43:364-75. 